\title{
A 17-years-old Girl Presenting with Spontaneous Cerebrospinal Fluid Rhinorrhea Diagnosed as Aqueductal Stenosis: A Case Report
}

\author{
Umma Salma ${ }^{1}$, Mohammad Abdus Sattar Sarker ${ }^{2}$, Abed Hossain Khan ${ }^{3}$, Nahida Zafrin ${ }^{4}$, MA Jalil Chowdhury. \\ ${ }^{1}$ Assistant Professor (Medicine), Dhaka National Medical College and Hospital ${ }^{2}$ Assistant Professor (Medicine), Sir Salimullah Medical College \\ ${ }^{3}$ Medical Officer, Medicine, BSSMU ${ }^{4}$ Clinical Pathologist, Sylhet MAG Osmani Medical College. ${ }^{5}$ Professor and Chairman of Internal Medicine, \\ BSSMU
}

\begin{abstract}
:
A 17-year-old girl presented with chronic headache and spontaneous CSF rhinorrhea. She had no history of head trauma. MRI of her brain showed aqueductal stenosis associated with triventriculomegaly with partially empty sella. Following insertion of ventriculo peritoneal shunt, her headache and CSF rhinorrhea completely recovered. Though very uncommon but aqueductal stenosis should be kept as a differential diagnosis for etiology of chronic CSF rhinorrhea.
\end{abstract}

Keyword: cerebrospinal fluid rhinorrhea, aqueductal stenosis

[BSMMU J 2014; 7 (2) : 134-137]

\section{Introduction :}

Aqueductal stenosis is a rare cause of CSF rhinorrhea. There are several potential causes of aqueductal stenosis such as congenital, post infection or post haemorrhage, idiopathic, acquired (some patient present in adulthood with acute or gradual onset of hydrocephalus), tumor associated with non communicating hydrocephalus. Aqueductal stenosis usually manifests in infancy but is uncommon in adulthood. In infancy usually manifestations are failure to thrive and/or bulging frontanelle, whereas in adulthood it manifests as raised intracranial pressure symptom. The treatment of these cases is widely variable. In this article we are reporting an adult case of aqueductal stenosis which manifested as spontaneous CSF rhinorrhea without any history of head trauma. Spontaneous CSF rhinorrhea is uncommon as primary presenting feature of aqueductal stenosis, with only 10 reported cases worldwide 1-10. Probably this is the first reported case in Bangladesh.

\section{Case Report}

A 17-year-old college girl got herself admitted in Dhaka

Address for Correspondence: Dr. Umma Salma Assistant Professor, Dhaka National Medical College Cell phone: 01727346271
National Medical College (DNMC) with recurrent episode of severe headache for last five years. Headache used to occur once or twice a month, not associated with vomiting or photophobia. She also complained of watery discharge from her left nostrils for last three years. Her nasal discharge was profuse, watery and accentuated by coughing and bending forward. Her headache and discharge had no diurnal variations. There was no history of fever, weight loss, anorexia or history suggestive of tuberculosis. She never experienced visual obscuration, convulsion, vomiting or unconsciousness. She had no history of head trauma. On examination she was conscious, oriented, having normal higher psychic function, all the cranial nerves were normal. On opthalmoscopy there was no papilloedema. There were no motor, sensory or cerebellar signs and no sign of meningitis.

Her investigations showed CBC normal, ESR $20 \mathrm{~mm}$ in first hour, Random Blood Sugar 120mg/dl, serum urea, serum creatinine, serum electrolyte, CXR P/A view normal. Sputum for Acid Fast Bacilli negative, Mantoux test negative. CT scan of brain showed triventricular moderate obstructive hydrocephalus (Fig-1). MRI brain showed evidence of aqueductal stenosis with gross triven- 
ticular hydrocephalus with partially empty sella (Fig2a,b). Biochemical analysis of nasal discharge was consistent with cerebrospinal fluid (CSF). Fundal photograph was normal. Visual field analysis showed arcuate scotoma in the right eye and paracentral scotoma in the left eye.

After giving some conservative treatment the patient was referred to Bangabandhu Sheikh Mujib Medical University (BSMMU) for further management. There, after further assessment, ventriculo peritoneal shunt was inserted. After surgery the patient was asymptomatic and recovered from previous symptoms of headache and rhinorrhea.

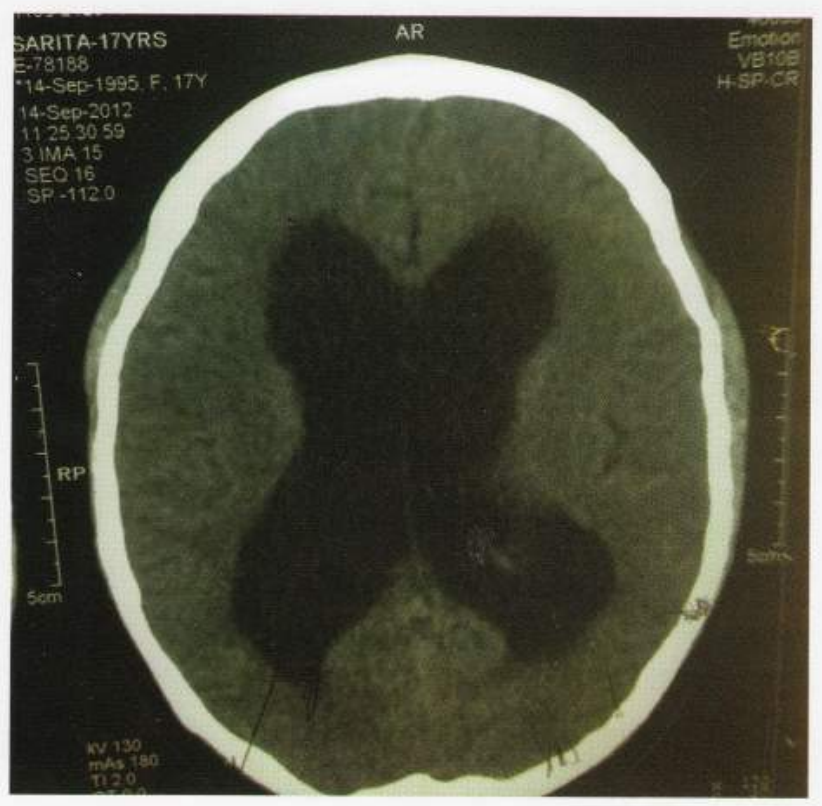

Fig 1: CT Scan of head showing hydrocephalus

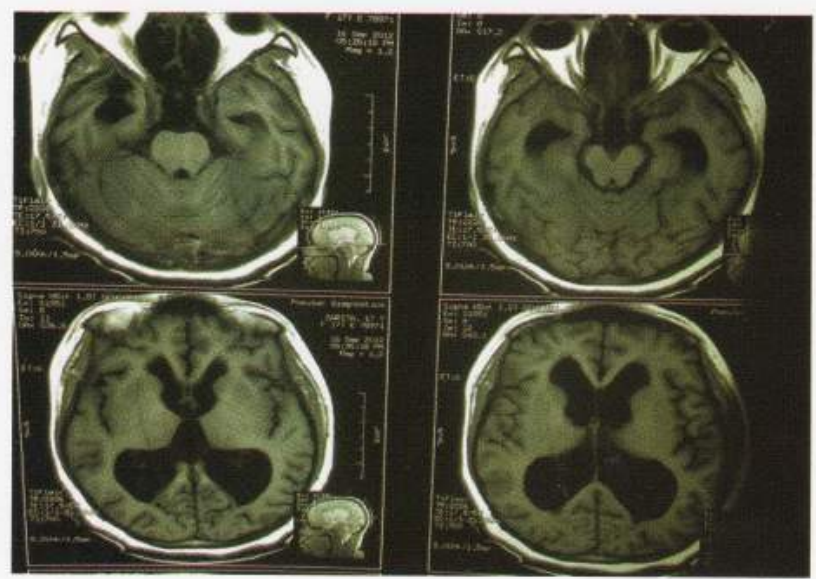

Fig-2a: MRI of brain showing triventricular hydrocephalus with evidence of aquductal stenosis

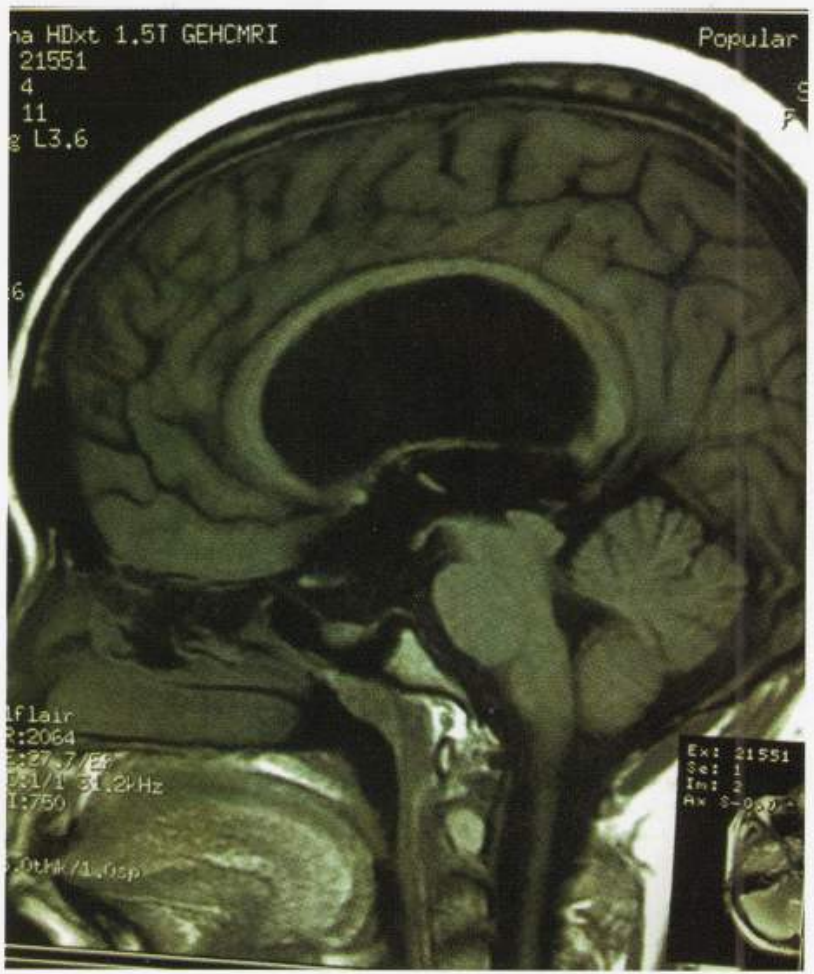

Fig-2b: MRI of brain showing partilally empty sella

\section{Discussion :}

CSF rhinorrhea commonly follows head trauma (Traumatic CSF rhinorrhea). Non-traumatic (Spontaneous) CSF rhinorrhea which is less prevalent usually associated with normal or high CSF pressure. Causes of normal pressure CSF leakage are related to dural disruption following intracranial surgery, osteomyelitis of skull and paranasal sinuses, congenital abnormalities of the brain with defect of the adjacent membrane and surrounding bone (example: naso-meningoencephaloceles) and destructive neoplasms at the base of the skull. High pressure CSF rhinorrhea is caused by intracranial benign and malignant tumors ${ }^{7,11-13}$ or associated with hydrocephalus. Pituitary tumors cause erosion of sella turcica floor and are frequently associated with spontaneous CSF rhinorrhea. ${ }^{14,15}$

Spontaneous CSF rhinorrhea associated with aqueductal stenosis can occur in various ways. The cribriform plate is an anatomically fragile portion of the anterior cranial fossa base, vulnerable to the normal rhythmic variation in 
the CSF pressure. This relatively weak site can allow transmission of CSF from the intracranial region to the nasal cavity, presumably related to constitutional or developmental factors. Anatomical studies of embryological variations in the cribriform plate and the traversing olfactory fibers have identified incompletely occluded holes around the olfactory fibers in the cribiform plate, ${ }^{16}$ persistent cranial pharyngeal canal, ${ }^{17}$ fistula in the retained embryonic lumen of the olfactory bulb, and meningeal dysplasia in the region of olfactory nerve. ${ }^{18}$ Any act which increases intracranial pressure suddenly such as sneezing, coughing could open previously occult anatomical defect and allow CSF leakage. ${ }^{19}$

In young people space occupying lesion is a common cause of triventriculomegaly. In our case triventriculomegaly was unusually secondary to aquiductal stenosis. Contrast enhanced MRI exclude any tumor in the brain. Longstanding persistent or intermittent elevated intracranial pressure secondary to aquiductal stenosis could have primarily resulted in the thinning and erosion of the floor of the anterior cranial fossa. Spontaneous CSF rhinorrhea may act as a natural vent or protective mechanism to prevent dangerous elevation of intracranial pressure. This explains normal opthalmoscopy finding of our patient in spite of having hydrocephalus and development of headache as a feature of raised ICP developed earlier than rhinorrhea coincides with the common presentation. A review of chronological relationship of CSF rhinorrhea with respect to signs or symptoms of increased intracranial pressure in 19 cases revealed that increase intracranial pressure preceded the onset of rhinorrhea in 16 cases. Rhinorrhea alone was the presenting symptom in only 3 patients. In 1 instance rhinorrhea and symptoms of raised intracranial pressure (ICP) developed simultaneously. ${ }^{8}$

In aqueductal stenosis direct communication between the lateral ventricle and the frontal sinuses may cause CSF rhinorrhea ${ }^{1}$ Three cases of the aqueductal stenosis have been reported manifesting as a fistulas communication between the protruding frontal lobe and the defect in the cribiform plate..$^{6,78}$ A case of aqueductal stenosis presented with spontaneous CSF rhiniorrea associated with the empty sella syndrome. ${ }^{4}$ In our case there was no fistulas communication demonstrable between the brain and paranasal sinuses. This probably was responsible for the immediate and complete cessation of spontaneous CSF rhinorrhea following the ventriculoperitoneal shunt insertion.

The management of spontaneous high pressure CSF rhinorrhea is directed towards the primary pathology. Performance of a CSF diversion procedure only may be sufficient. Surgical repair of the CSF fistula is mandatory if there is persistent CSF rhinorrhea following the CSF diversion procedure, and is also warranted in patients who develop tension pneumocephalus after ventricularperitoneal (VP) shunting. ${ }^{2,3,5}$ Multilayered reconstruction of the anterior cranial fossa using frontal muscle flap, pericranial and temporalis muscle-fascia based flap, or a free vascularized omental flap is necessary. ${ }^{20-22}$ Endoscopic repair of CSF fistula has been successful with a low complication rate. ${ }^{23}$ In our patient the insertion of VP shunt stopped the CSF rhinorrhea permanently and hence no direct repair of the fistula was necessary.

Spontaneous CSF rhinorrhea is rare as a primary presenting syndrome of aqueductal stenosis. Longstanding spontaneous CSF rhinorrhea indicates the possibility of a concurrent intracranial pathology. The diagnosis of aqueductal stenosis although uncommon should be considered in such situation.

\section{References :}

1. Cabezudo JM, Vaquero J, Garcia-de-Sola R, Areitio E, Martinez R. Direct communication between the lateral ventricle and the frontal sinus as the cause of CSF rhinorrhea in aqueductal stenosis. Acta Neurochir (Wien) 1981; 57: 95-98.

2. Ikeda K, Nakano M, Tani E. Tension pneumocephalus complicating ventriculoperitoneal shunt for cerebrospinal fluid rhinorrhoea: case report. J Neurol Neurosurg Psychiatry 1978; 41: 319-322.

3. Jooma R, Grant DN. Cerebrospinal fluid rhinorrhea and intraventricular pneumocephalus due to inter- mittent shunt obstruction. Surg Neurol 1983; 20: 231-234.

4. Kushner J, Meschan I, Alexander E Jr. Cerebrospinal fluid rhinorrhea, aqueductal stenosis, and the empty sella. Md State Med J 1973; 22: 51-54. 
5. Little JR, MacCarty CS. Tension pneumocephalus after insertion of ventriculoperitoneal shunt for aqueductal stenosis. J Neurosurg 1976; 44: 383-385.

6. Nishikawa M, Karasawa J, Kamada K, Kikuchi H, Makita Y. Non-traumatic cerebrospinal fluid rhinorrhea associated with aqueductal obstruction. No To Shinkei 1972; 24: 173-177.

7. Rovit RL, Schechter MM, Nelson K. Spontaneous high-pressure cerebrospinal rhinorrhea due to lesions obstructing flow of cerebrospinal fluid. J Neurosurg 1969; 30: 406-412.

8. Schechter MM, Rovit RL, Nelson K. Spontaneous high pressure CSF rhinorrhoea: Cerebrospinal fluid leakage caused by long-standing increased intracranial pressure. Br J Radiol 1969; 42: 619-622.

9. Tokuno T, Ban S, Nakazawa K, Yoshida S, Matsumoto S et al. Non - traumatic cerebrospinal fluid rhinorrhea associated with hydrocephalus: a case report. No Shinkei Geka 1995; 23: 265-269.

10. Muzumdar D, Nadkarni T, Goel A. Spontaneous cerebrospinal fluid rhinorrhea as a presenting symptom of aqueductal stenosis-case report. Neurol Med Chir 2003;43:626-9.

11. Kane PJ, Mendelow AD, Keogh AJ, Symon L. Cerebrospinal fluid rhinorrhea associated with colloid cyst. Br J Neurosurg 1991; 5: 317-320.

12. Morley TP, Hetherington RF. Traumatic cerebrospinal fluid rhinorrhea and otorrhea, pneumocephalus and meningitis. Surg Gynecol Obstet 1957; 104: 88-98.

13. Vaquero J, Cabezudo JM, Salazar AR, Brasa J. Symptomatic intrasphenoidal meningoencephalocele after removal of a parasagittal meningioma. Acta Neurochir (Wien)1981; 57: 61-65.
14. Hanel RA, Prevedello DM, Correa A, Antoniuk A, Araujo JC. Cerebrospinal fluid fistula as the presenting manifestation of pituitary adenoma: case report with a 4-year follow-up. Arq Neuropsychiatry 2001; 59:263-265.

15. Norsa L. Cerebrospinal rhinorrhea with pituitary tumors. Neurology $1965 ; 13: 864-868$.

16. Locke CE. The spontaneous escape of cerebrospinal fluid through the nose: Its occurrence with brain tumors. Arch Neurol Psychiatry $1926 ; 15: 309$.

17. Loftus JE. Cerebrospinal fluid rhinorrhoea with report of a case. Laryngoscope 1923; 33: 617-632.

18. Rand RW. Spontaneous cerebrospinal fluid rhinorrhea. Bull Los Angeles Neurol Soc 1953; 18: 74-83.

19. O'Connell JEA. Primary spontaneous cerebrospinal fluid rhinorrhea. J Neurol Neurosurg Psychiatry 1964; 27: 241-246.

20. Costa H, Cunha C, Conde A, Cerejo A, Baptista A et al. Reconstruction of the anterior cranial base with a frontal muscle flap in cerebrospinal fluid fistulae. Acta Med Port 1997; 10: 19-26.

21. Goel A. Multilayer reconstruction of the anterior cranial fossa floor. Br J Neurosurg 1998; 12: 254-258.

22. Normington EY, Papay FA, Yetman RJ. Treatment of recurrent cerebrospinal fluid rhinorrhea with a free vascularized omental flap: a case report. Plast Reconstr Surg 1998; 98: 514-519.

23. Ramsden JD, Corbridge R, Bates G. Bilateral cerebrospinal fluid rhinorrhoea. J Laryngol Otol 2000; 114: 137-138 